\title{
Auto-steered Information-Decision Processes for Electric System Asset Management
}

\author{
James D. McCalley, Vasant G. Honavar, Sarah M. Ryan, William Q. Meeker, \\ Ronald A. Roberts, Daji Qiao, and Yuan Li \\ Iowa State University, Ames, IA 50011, US \\ $\{j d m$, honavar, smryan, wqmeeker, rroberts, daji, \\ tua\} @iastate.edu
}

\begin{abstract}
The total replacement value of the US transmission lines alone (excluding land) is conservatively estimated at over $\$ 100$ billion dollars [1] and triples when including transformers and circuit breakers. Investment in new transmission equipment has significantly declined over the past 15 years. Some of the equipment is well beyond intended life, yet is operated under increasing stress, as load growth, new generation, and economically motivated transmission flows push equipment beyond nameplate limits. Maintaining acceptable electric transmission system reliability and delivering electric energy at low energy prices requires innovations in sensing, diagnostics, communications, data management, processing, algorithms, risk assessment, decision-making (for operations, maintenance, and planning), and process coordination. This paper overviews a comprehensive approach to develop methods and processes in these areas, driven by the ultimate objective to develop a hardware-software prototype capable of auto-steering the information-decision cycles inherent to managing operations, maintenance, and planning of the high-voltage electric power transmission systems.
\end{abstract}

\section{Introduction}

In electric power transmission systems, the assets include transmission lines, support structures, transformers, power plants, and protection equipment. Condition information includes loading or operating histories, inspection data, periodic and asneeded testing and diagnostic results, and continuous diagnostic measurements, the latter of which are typically collected via intelligent electronic devices (IED) and stored within substation servers. A single transmission company, each of which has their own centralized control center, has responsibility for many thousands of each equipment type. A single control area, represented by an Independent System Operator (ISO), oversees and coordinates activities of a number of different transmission companies. The eastern and western US interconnections are each comprised of a number of ISOs; the only other US interconnection, Texas, has only one. Failure of an asset may affect physical and economic performance of the entire interconnection and always increases likelihood of additional failures. Because economic performance (power supply allocation among power plants) affects transmission loading which affects failure likelihood and consequence, operational 
risk-reduction inevitably results in less economic power supply. Frequency and severity of blackout scenarios as observed on August 14, 2003 are affected by policies associated with equipment operation, maintenance, and planning.

The objective in this work is to develop a hardware-software prototype capable of auto-steering the information-decision cycles inherent to managing operations, maintenance, and planning of the high-voltage electric power transmission systems. We focus on the needs of the most critical electric transmission equipment, including power transformers, circuit breakers, and transmission lines. Similar equipment exists at the distribution level, so the work will find direct application there. Figure 1 illustrates the structure of the problem and facilitates description of how we intend to approach its solution. We overview intended implementation of the 5 different layers in what follows.

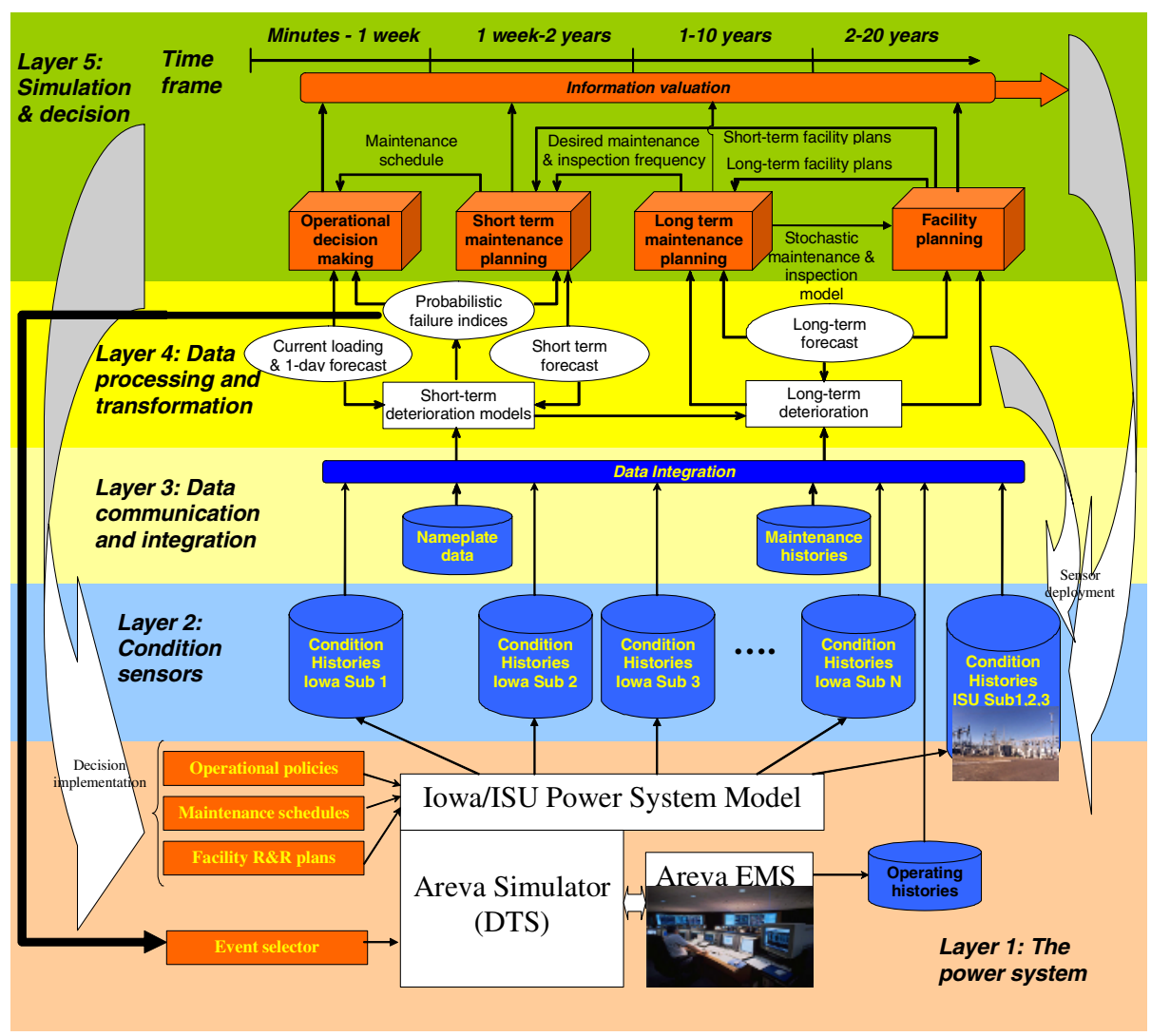

Fig. 1. The structure of the asset management problem

Layer 1, The power system: The prototype will center on a continuously running model of the Iowa power system using network data provided by local utility companies using a commercial-grade (Areva) simulator. 
Layer 2, Condition sensors: As indicated by the taller "Condition History" cylinder at the far right of layer, 3 campus substations will be equipped with sensors, communication equipment, and servers to provide a benchmark prototype for hardware implementation. Other substations will be represented virtually, each with its own unique database containing the condition data for that substation provided by the utility company.

Layer 3, Data communication and integration: This will entail intra-substation communication using wireless between IEDs and substation server together with federated data integration to provide efficient, dependable, and secure mechanisms for interfacing Layer 4 data transformation algorithms with the data resources.

Layer 4, Data processing and transformation: This layer will operate on the integrated data from layer 3 to produce, for each component/failure mode/time, an estimate of that particular component/failure mode deterioration level at the given time. This will require deterioration models, and we target such models for the chemical degradation processes in oil and cellulose (both of which provide insulation in power transformers). We will also need stochastic models to predict future degradation, and we further describe these models in Section 2.

Layer 5, Simulation and decision: This layer will utilize the component probabilistic failure indices from layer 4 together with short and long-term system forecasts to drive integrated stochastic simulation and decision models. These models will operate interactively, so that simulation and decision in each time frame utilizes information from simulation and decision within other time frames. Resulting operational policies, maintenance schedules, and facility reinforcement plans will then be implemented on the power system (as represented by the Areva simulator). The decision models will also be used to discover the value of additional information. This valuation will be used to drive the deployment of new sensors and redeployment of existing sensors, impacting Layer 2. This layer is further described in Section 3.

\section{Layer 4: Data Processing and Transformation}

Component condition, deterioration level, or propensity to fail, is essential information for asset management decision problems. Our objective in this part of the work is to develop methods of computing component (or subsystem) failure probabilities. One unique aspect of this work is that in addition to steady-state failure probabilities that capture average behavior over a large number of components and over an extended period of time, we also require transient failure probabilities to capture instantaneous behavior for each specific component.

Consider a set of condition vectors $\mathrm{c}(\mathrm{t})=\left[\mathrm{c}_{1}(\mathrm{t}), \mathrm{c}_{2}(\mathrm{t}), \ldots, \mathrm{c}_{\mathrm{K}}(\mathrm{t})\right]$ for $\mathrm{K}$ similar components taken over an extended period of time $t=0,1, \ldots, T$, where each vector $c_{k}(t)$ provides $\mathrm{M}$ different measurements $\mathrm{c}_{\mathrm{k} 1}(\mathrm{t}), \mathrm{c}_{\mathrm{k} 2}(\mathrm{t}), \ldots \mathrm{c}_{\mathrm{kM}}(\mathrm{t})$, on component $\mathrm{k}$ characterizing its condition at time $t$. The total possible number of measurements is less than $\mathrm{K} \times \mathrm{T} \times \mathrm{M}$ because there are different frequencies for which different measurements are taken. We will augment $\mathrm{c}(\mathrm{t})$ with operational and environmental information in building predictive failure models. For some system components, failure is closely related to a single condition measurement that can be measured over time and modeled in a manner that allows reasonably accurate prediction of failure 
(e.g., extent of vegetation growth or the amount of chemical degradation). Let $c(t, e ; \beta)$ denote the expected level of degradation for a unit subjected to environmental conditions e, where $\beta$ is a vector of unknown model parameters to be estimated from available data. The form of the function $\mathrm{c}$ may be suggested by physical-chemical theory, (see, for example, $[2,3,4]$ ), past experience, or the available data. A failuretime cdf $F(t)$ is induced by a specified model for $c(t, e ; \beta)$, the environment $e$, and a definition of failure (usually a specified value $c_{\mathrm{f}}$, beyond which failure is said to have occurred). Stochastic behavior in $\mathrm{c}(\mathrm{t}, \mathrm{e} ; \beta)$ can be captured either by using a stochastic process model (e.g., [5]) or by driving a deterministic model with a stochastic environmental model (e.g., [6]). As new condition information is received for a given unit, it is possible to update the failure probability for that unit. For the special case in which all units are in a common and constant environment, [7] develops a model to describe the effect that nondestructive inspections will have on the failure probability. It is possible to generalize this "degradation analysis" approach to a vector of condition measurements, but statistical modeling of the joint distribution of a vector of condition measurements is more difficult, especially if the dimension exceeds 2 . The Markov modeling approaches discussed next provide a useful alternative.

We can often characterize boundary conditions separating $\mathbf{J}$ states of deterioration in component $\mathrm{k}$ in terms of the measurements $c_{k}(t)$, via a deterioration function $\mathrm{g}\left[\mathrm{c}_{\mathrm{k}}(\mathrm{t})\right]$. The deterioration function returns a deterioration level $\mathrm{j}$ identified by $\mathrm{d}_{\mathrm{j}-}$ ${ }_{1}<\mathrm{g}\left[\mathrm{c}_{\mathrm{k}}(\mathrm{t})\right]<\mathrm{d}_{\mathrm{j}}$, where the last state $\mathrm{j}=\mathrm{J}$ represents the failed state. State $\mathrm{J}$ need not represent the rare "blue smoke" condition where the component has catastrophically failed (and for which little data is typically available). Rather, state J represents a set of measurement values for which engineering judgment indicates the component should be removed from service. This approach to computing failure probabilities is illustrated in Fig. 2, based on multistate Markov models, where each of $\mathbf{J}$ states is represented as a deterioration level. The representation of Fig. 2 shows $\mathrm{J}=4$ deterioration levels, and deterioration level $\mathrm{j}$ is reached only from deterioration level $\mathrm{j}-1$. Yet, the model is flexible; any number of deterioration levels can be represented, and, if data indicates transitions occur between non-consecutive states (e.g., 1 to 3), the model can accommodate this.

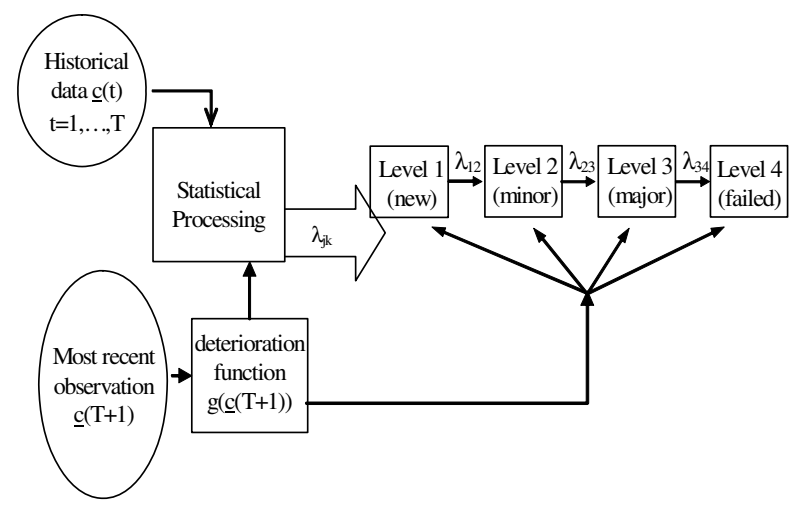

Fig. 2. Computing contingency probability reductions 
The model parameters capture the deterioration in equipment state as influenced by past loading and environmental conditions. To capture future effects of variation in such conditions on model parameters, one needs to model the dependency of the transition intensities on these parameters. To account for uncertainty in state identification through observation or indirect measurement, the variance of the conditional probability of observation given the state, referred to as $\sigma_{\mathrm{ki}}$ for component $\mathrm{k}$, state $\mathrm{i}$ is used. This parameter can also facilitate the analysis to identify investments to make for obtaining better or more information, described in Section 3.2.

Once transition intensities are determined, state probabilities are obtained from the transition probability matrix and initial state vector. We denote this failure probability for the $\mathrm{k}^{\text {th }}$ component as $\mathrm{p}_{\mathrm{k}}(\mathrm{c})$, a function of the time-dependent physical condition of the equipment $\mathrm{c}(\mathrm{t})$. This modeling provides the ability to predict the effect that maintenance will have on failure probability and expected time to failure, metrics that are important for a number of decision problems. The expected time to failure is captured by computing first passage times $[8,9]$.

\section{Layer 5: Simulation, Decision and Information Valuation}

Asset management decision problems are characterized by: (1) strong interdependencies between physical performance of individual assets, physical performance of the overall system, and economic system performance; (2) limited resources; (3) important uncertainties in individual component performance, system loading conditions, and available resources; (4) multiple objectives. We describe these decision problems in this section, together with our intent to solve them in an integrated fashion.

\subsection{Simulation and Decision}

Asset management decision problems can be classified into one of 4 types which all involve resource allocation with the objective to minimize cost and risk. These specific asset management decision problems include (a) Operations, (b) Short-term maintenance selection and scheduling, (c) Long-term maintenance planning, and (d) Facility planning.

These problems differ primarily in their time scale but are linked by a common focus on the interactions between the condition of equipment and the decisions taken. The operational decision problem of how to meet demand in the next hour to week treats facilities available and their deterioration levels as given (though the deterioration is not known precisely). The contribution here is to use condition measurements to more accurately estimate short-term failure probabilities along with the deterioration effects of loading each piece of equipment at various levels, and to integrate these improved estimates into the dispatch and unit commitment decisions. The tactical decision problem to allocate resources for maintenance in the next 6-24 months suppresses detail about hourly operations but considers an aggregate description of equipment loading when deciding how to allocate resources to best manage the condition of the equipment. Our approach will use historical data to better judge the combined effects of maintenance and loading on the equipment 
deterioration and use this information to improve maintenance scheduling. The longterm maintenance problem examines tradeoff between maintenance expense and equipment life to find inspection and maintenance policy to minimize expected long run cost of keeping the equipment in service reliably. The strategic decision problem to both expand capacity and replace equipment over the next 2-20 years takes as input distributions of equipment life lengths resulting from adopted maintenance policies and determines when to replace existing equipment and invest in additional assets. Unlike previous models for equipment replacement and capacity expansion, we consider the cumulative effect of power flow on equipment life and take advantage of better data-driven life length predictions.

When we integrate these optimization problems having different time-scales together, we will treat the quantities that vary much more slowly as static and model quantities that vary much faster in a way that ignores the details of their variations, such as by replacing fast-moving quantities by their averages. A similar strategy is used in hierarchical planning of manufacturing systems [10].

\subsection{Information Valuation and Sensor Deployment}

A last but critical decision problem to be addressed is how to extract from data transformation (Layer 4) and decision (Layer 5) algorithms identification of economically sound opportunities for obtaining better information, thereby reducing uncertainty and improving decision-making capability. A simple case is when an abrupt measurement change causes immediate suspicion that a failure is imminent. The response is to inspect the equipment. Additional more specialized measurements may be done, and if those measurements confirm a problem, the equipment is removed. Such situations are addressed via alarms. A decision to obtain information is clear in this case, because imminent failure poses high capital loss and physical harm to humans.

Decision to gather information is more difficult for maintenance and planning problems because the payoff (or avoided loss) is not so pronounced. We address this problem via a two-stage information valuation approach $[11,12,13,14]$. In the first stage, we determine candidate components for which additional information may be of interest. Denoting the value of the objective function at the solution as $\Gamma$, we compute an index giving sensitivity in $\Gamma$ due to component $\mathrm{k}$, as $\sigma_{\mathrm{ki}}\left(\partial \Gamma / \partial \mathrm{p}_{\mathrm{k}}\right)$ where $\mathrm{p}_{\mathrm{k}}$ is the failure probability of component $\mathrm{k}$ and $\sigma_{\mathrm{ki}}$ (see end of Section 2) is the deviation in the observation for component $\mathrm{k}$ given that it is in state $\mathrm{i}$. Components having high index are candidates to consider in the information valuation stage. Other selection criteria can be considered, e.g., we could identify components that are almost or barely selected by the decision algorithm. We denote additional information associated with candidate component $\mathrm{k}$ as $\mathrm{r}_{\mathrm{km}}$, which indicates component $\mathrm{k}$ is in state $\mathrm{m}$. Such information may be obtained by installing more or better sensors at a cost. For example, a 50 year-old transformer that is a clear candidate for replacement may be operating with no monitoring equipment, yet installation of such equipment, providing information $\mathrm{r}_{\mathrm{km}}$, may result in decision to operate the unit for more years.

Following [13], states in which component $\mathrm{k}$ may reside are identified by $\mathrm{i}=\{1, \ldots, \mathrm{S}\}$, with each state having probability $\pi_{\mathrm{i}}$ obtained from procedures described in Section 2. Simulation and decision algorithms are then repeated once for each 
possible state of component $\mathrm{k}$, generating solutions with corresponding objective values. Denote identified solutions (alternatives) by $a=\{1, \ldots, A\}$. Thus, for each combination of state and alternative we have a consequence $c(a, i)$. Denoting the utility of an alternative as $\mathrm{u}(\mathrm{a})$ and of a consequence as $\mathrm{v}(\mathrm{c})$, we desire to choose the alternative to maximize expected utility $\mathrm{u}(\mathrm{a}, \pi)=\sum_{\mathrm{i}=1, \mathrm{~S}}\left[\pi_{\mathrm{i}} \mathrm{v}(\mathrm{c}(\mathrm{a}, \mathrm{i}))\right]$. The decision to obtain additional information is based on expected utility gains from shifting to better choices among the set of actions. Denote $\mathrm{a}_{0}$ as the optimal alternative with no additional information, identified using prior probabilities $\pi_{\mathrm{i}}$, and $\mathrm{a}_{\mathrm{m}}$ as the optimal alternative with the additional information $r_{k m}$. Then the value of information $r_{k m}$ is given by $\Delta\left(\mathrm{r}_{\mathrm{km}}\right)=\mathrm{u}\left(\mathrm{a}_{\mathrm{m}}, \pi_{\mathrm{i}, \mathrm{m}}\right)-\mathrm{u}\left(\mathrm{a}_{0}, \pi_{\mathrm{i}, \mathrm{m}}\right)=\sum_{\mathrm{i}=1, \mathrm{~S}}\left[\pi_{\mathrm{i}, \mathrm{m}} \mathrm{v}\left(\mathrm{c}\left(\mathrm{a}_{0}, \mathrm{i}\right)\right)\right]-\sum_{\mathrm{i}=1, \mathrm{~S}}\left[\pi_{\mathrm{i}, \mathrm{m}} \mathrm{v}\left(\mathrm{c}\left(\mathrm{a}_{\mathrm{m}}, \mathrm{i}\right)\right)\right]$, where the posterior probabilities $\pi_{\mathrm{i}, \mathrm{m}}$ are given by $\pi_{\mathrm{i}, \mathrm{m}}=\operatorname{Pr}\left\{\mathrm{r}_{\mathrm{km}} \mid \mathrm{i}\right\} \pi_{\mathrm{i}} / \operatorname{Pr}\left\{\mathrm{r}_{\mathrm{km}}\right\}$. However, the decision to seek the additional information must be done ex-ante to be useful, and so

\begin{tabular}{|c|c|c|c|c|c|c|c|}
\hline Component state & Good & Fair & Weak & Fail & & & \\
\hline Ideal measure & 0 & 1 & 0 & 0 & \multirow{4}{*}{$\begin{array}{l}\text { Simulation } \\
\& \text { decision } \\
\text { model }\end{array}$} & True decision & \multirow{4}{*}{$\begin{array}{l}\text { Decision } \\
\text { utility } \\
\text { estimation }\end{array}$} \\
\hline Measure $(1)^{*}$ & 0.2 & 0.4 & 0.2 & 0.2 & & Decision (1) & \\
\hline$\ldots$ & $\ldots$ & $\ldots$ & $\ldots$ & $\ldots$ & & $\ldots$ & \\
\hline Measure (n) & 0.1 & 0.7 & 0.1 & 0.1 & & Decision (n) & \\
\hline
\end{tabular}

* Current measurement.

Fig. 3. Illustration of information valuation and sensor deployment

we cannot know that we will obtain $\mathrm{r}_{\mathrm{km}}$, i.e., that we will learn that component $\mathrm{k}$ is in state $\mathrm{m}$. But we can assess (subjectively, or from historical data) the probability of learning from the new information that the component is in state $\mathrm{m}$, which is $\operatorname{Pr}\left\{\mathrm{r}_{\mathrm{km}}\right\}$. Then we may compute the expectation of the value associated with the new information as $\mathrm{E}\left\{\Delta\left(\mathrm{r}_{\mathrm{km}}\right)\right\}=\sum_{\mathrm{m}=1, \mathrm{~S}} \operatorname{Pr}\left\{\mathrm{r}_{\mathrm{km}}\right\}\left[\mathrm{u}\left(\mathrm{a}_{\mathrm{m}}, \pi_{\mathrm{i}, \mathrm{m}}\right)-\mathrm{u}\left(\mathrm{a}_{0}, \pi_{\mathrm{i}, \mathrm{m}}\right)\right]$. We will use this approach to interface with Layers 4 and 5 procedures for assessing where and when to obtain additional information.

\section{Conclusions}

This paper gives a framework of a hardware-software prototype capable of autosteering the information-decision cycles inherent to managing operations, maintenance, and planning of the high-voltage electric power transmission systems. The framework is divided into 5 layers and described in this paper accordingly. Although each layer represents an essential and substantive part of the framework, the paper focuses on the data transformation (in layer 4) and decision (in layer 5) elements. 


\section{Acknowledgments}

The work described in this paper is funded by the National Science Foundation under grant NSF CNS0540293.

\section{References}

1. M. Gallaher, S. Johnston, B. Kirby, "Changing measurement and standards needs in a deregulated electric utility industry," Nov., 1999, Research Triangle Institute, http://www.rti.org/pubs/dereg.pdf.

2. Lu, C.J., and Meeker, W.Q. (1993), Using Degradation Measures to Estimate a Time-toFailure Distribution. Technometrics 35, 161-174.

3. Meeker, W.Q., and LuValle, M.J. (1995), An Accelerated Life Test Model Based on Reliability Kinetics. Technometrics 37, 133-146.

4. Meeker, W.Q., Escobar, L.A., Lu, C.J. (1998), Accelerated Degradation Tests: Modeling and Analysis. Technometrics 40, 89-99.

5. Grall, A., Dieulle, L., Berenguer, C., and Roussignol, M. (2002), Continuous-Time Predictive-Maintenance Scheduling for a Deteriorating System, IEEE Transactions on Reliability 51, 141-150.

6. Meeker, W. Q., Escobar, L. A., and Chan, V. (2002), Using Accelerated Tests to Predict Service Life in Highly-Variable Environments. Chapter 19 in Service Life Prediction Methodology and Metrologies Washington: American Chemical Society, J. W. Martin and D. R. Bauer, Editors.

7. Garrigoux, C.G. and Meeker, W.Q. (1995), Assessing the Effect of In-Service Inspections on the Reliability of Degrading Components, Recent Advances in Life-Testing and Reliability, N. Balakrishnan (Editor), CRC Press.

8. G. Anders, Probability Concepts in Electric Power Systems, John Wiley, New York, 1990.

9. A. Leite da Silva and J. Endrenyi, "Application of First Passage Times in the Markov Representation of Electric Power Systems," Proceedings of the 4th International Conference on Probabilistic Methods Applied to Power Systems, Rio de Janeiro, Brazil, Sept. 1994.

10. S. B. Gershwin, "Hierarchical Flow Control: A Framework for Scheduling and Planning Discrete Events in Manufacturing Systems," Proceedings of the IEEE Volume 77, Issue 1, Jan. 1989 Page(s):195-209.

11. H. Raiffa, "Decision analysis: Introductory lectures on choices under uncertainty," Addison-Wesley, 1968.

12. J. Mnarschak, "Economic information, decision, and prediction," Volume II, D. Reidel Publishing Co., 1974.

13. A. Baker, "Business decision-making," Croom-Helm, 1981.

14. J. Hirshleifer, "Time, Uncertainty, and Information," Basil Blackwell, 1989. 